IJMMS 27:5 (2001) 261-267

PII. S0161171201010778

http://ijmms.hindawi.com

(c) Hindawi Publishing Corp.

\title{
INTUITIONISTIC FUZZY INTERIOR IDEALS OF SEMIGROUPS
}

\author{
KYUNG HO KIM and YOUNG BAE JUN
}

(Received 18 August 2000 and in revised form 11 December 2000)

\begin{abstract}
We consider the intuitionistic fuzzification of the concept of interior ideals in a semigroup $S$, and investigate some properties of such ideals. For any homomorphism $f$ from a semigroup $S$ to a semigroup $T$, if $B=\left(\mu_{B}, \gamma_{B}\right)$ is an intuitionistic fuzzy interior ideal of $T$, then the preimage $f^{-1}(B)=\left(f^{-1}\left(\mu_{B}\right), f^{-1}\left(\gamma_{B}\right)\right)$ of $B$ under $f$ is an intuitionistic fuzzy interior ideal of $S$.
\end{abstract}

2000 Mathematics Subject Classification. 20M12, 03E72, 94D05.

1. Introduction. The idea of "intuitionistic fuzzy set" was first published by Atanassov [1, 2], as a generalization of the notion of fuzzy set. Jun et al. considered the fuzzification of interior ideals in semigroups [3]. In this paper, we introduce the notion of an intuitionistic fuzzy interior ideal of a semigroup $S$, and then some related properties are investigated. Characterizations of intuitionistic fuzzy interior ideals are given. Also for any homomorphism $f$ from a semigroup $S$ to a semigroup $T$, if $B=\left(\mu_{B}, \gamma_{B}\right)$ is an intuitionistic fuzzy interior ideal of $T$, then the preimage $f^{-1}(B)=\left(f^{-1}\left(\mu_{B}\right), f^{-1}\left(\gamma_{B}\right)\right)$ of $B$ under $f$ is an intuitionistic fuzzy interior ideal of $S$.

2. Preliminaries. Let $X$ be a nonempty fixed set. An intuitionistic fuzzy set (IFS for short) $A$ is an object having the form

$$
A=\left\{\left(x, \mu_{A}(x), \gamma_{A}(x)\right): x \in X\right\}
$$

where the functions $\mu_{A}: X \rightarrow[0,1]$ and $\gamma_{A}: X \rightarrow[0,1]$ denote the degree of membership (namely $\mu_{A}(x)$ ) and the degree of nonmembership (namely $\gamma_{A}(x)$ ) of each element $x \in X$ to the set $A$, respectively, and $0 \leq \mu_{A}(x)+\gamma_{A}(x) \leq 1$ for all $x \in X$ (see Atanassov [1, 2]). For the sake of simplicity, we use the symbol $A=\left(\mu_{A}, \gamma_{A}\right)$ for the IFS $A=\left\{\left(x, \mu_{A}(x), \gamma_{A}(x)\right): x \in X\right\}$.

Let $S$ be a semigroup. By a subsemigroup of $S$ we mean a nonempty subset $A$ of $S$ such that $A^{2} \subseteq A$. A subsemigroup $A$ of a semigroup $S$ is called an interior ideal of $S$ if $S A S \subseteq A$. A mapping $f$ from a semigroup $S$ to a semigroup $T$ is called a homomorphism if $f(x y)=f(x) f(y)$ for all $x, y \in S$.

A fuzzy set $\mu$ in a semigroup $S$ is called a fuzzy subsemigroup of $S$ (see [3]) if $\mu(x y) \geq \mu(x) \wedge \mu(y)$ for all $x, y \in S$.

A fuzzy subsemigroup $\mu$ of a semigroup $S$ is called a fuzzy interior ideal of $S$ (see [3]) if $\mu(x a y) \geq \mu(a)$ for all $a, x, y \in S$. 
3. Intuitionistic fuzzy interior ideals. In what follows, $S$ denotes a semigroup unless otherwise specified.

DEFINITION 3.1. An IFS $A=\left(\mu_{A}, \gamma_{A}\right)$ in $S$ is called an intuitionistic fuzzy subsemigroup of $S$ if it satisfies

(IF1) $\mu_{A}(x y) \geq \mu_{A}(x) \wedge \mu_{A}(y)$,

(IF2) $\gamma_{A}(x y) \leq \gamma_{A}(x) \vee \gamma_{A}(y)$,

for all $x, y \in S$.

EXAMPLE 3.2. Let $S=\{0, e, f, a, b\}$ be a set with the following Cayley table:

\begin{tabular}{l|lllll}
\hline$\cdot$ & 0 & $e$ & $f$ & $a$ & $b$ \\
\hline 0 & 0 & 0 & 0 & 0 & 0 \\
$e$ & 0 & $e$ & 0 & $a$ & 0 \\
$f$ & 0 & 0 & $f$ & 0 & $b$ \\
$a$ & 0 & $a$ & 0 & 0 & $e$ \\
$b$ & 0 & 0 & $b$ & $f$ & 0 \\
\hline
\end{tabular}

Then $S$ is a semigroup (see [4]). Define an IFS $A=\left(\mu_{A}, \gamma_{A}\right)$ in $S$ by $\mu_{A}(0)=\mu_{A}(e)=$ $\mu_{A}(f)=1, \mu_{A}(a)=\mu_{A}(b)=0, \gamma_{A}(0)=\gamma_{A}(e)=\gamma_{A}(f)=0$, and $\gamma_{A}(a)=\gamma_{A}(b)=1$. By routine calculations we know that $A=\left(\mu_{A}, \gamma_{A}\right)$ is an intuitionistic fuzzy subsemigroup of $S$.

Definition 3.3. An intuitionistic fuzzy subsemigroup $A=\left(\mu_{A}, \gamma_{A}\right)$ of $S$ is called an intuitionistic fuzzy interior ideal of $S$ if

(IF3) $\mu_{A}(x a y) \geq \mu_{A}(a)$,

(IF4) $\gamma_{A}(x a y) \leq \gamma_{A}(a)$,

for all $x, y, a \in S$.

EXAMPLE 3.4. The IFS $A=\left(\mu_{A}, \gamma_{A}\right)$ in Example 3.2 is an intuitionistic fuzzy interior ideal of $S$.

THEOREM 3.5. If $\left\{A_{i}\right\}_{i \in \Lambda}$ is a family of intuitionistic fuzzy interior ideals of $S$, then $\cap A_{i}$ is an intuitionistic fuzzy interior ideal of $S$, where $\cap A_{i}=\left(\wedge \mu_{A_{i}}, \vee \gamma_{A_{i}}\right)$ and $\wedge \mu_{A_{i}}$ and $\vee \gamma_{A_{i}}$ are defined as follows:

$$
\begin{aligned}
& \wedge \mu_{A_{i}}(x)=\inf \left\{\mu_{A_{i}}(x) \mid i \in \Lambda, x \in S\right\}, \\
& \vee \gamma_{A_{i}}(x)=\sup \left\{\gamma_{A_{i}}(x) \mid i \in \Lambda, x \in S\right\} .
\end{aligned}
$$

Proof. Let $x, y, a \in S$. Then

$$
\begin{gathered}
\wedge \mu_{A_{i}}(x y) \geq \wedge\left(\mu_{A_{i}}(x) \wedge \mu_{A_{i}}(y)\right)=\left(\wedge \mu_{A_{i}}(x)\right) \wedge\left(\wedge \mu_{A_{i}}(y)\right), \\
\vee \gamma_{A_{i}}(x y) \leq \vee\left(\gamma_{A_{i}}(x) \vee \gamma_{A_{i}}(y)\right)=\left(\vee \gamma_{A_{i}}(x)\right) \vee\left(\vee \gamma_{A_{i}}(y)\right), \\
\wedge \mu_{A_{i}}(x a y) \geq \wedge \mu_{A_{i}}(a), \quad \vee \gamma_{A_{i}}(x a y) \leq \vee \gamma_{A_{i}}(a) .
\end{gathered}
$$

Hence $\cap A_{i}$ is an intuitionistic fuzzy interior ideal of $S$. 
THEOREM 3.6. If an IFS $A=\left(\mu_{A}, \gamma_{A}\right)$ in $S$ is an intuitionistic fuzzy interior ideal of $S$, then so is $\square A:=\left(\mu_{A}, \bar{\mu}_{A}\right), \bar{\mu}_{A}=1-\mu_{A}$.

Proof. It is sufficient to show that $\bar{\mu}_{A}$ satisfies conditions (IF2) and (IF4). For any $a, x, y \in S$, we have

$$
\begin{aligned}
\bar{\mu}_{A}(x y) & =1-\mu_{A}(x y) \leq 1-\left(\mu_{A}(x) \wedge \mu_{A}(y)\right) \\
& =\left(1-\mu_{A}(x)\right) \vee\left(1-\mu_{A}(y)\right)=\bar{\mu}_{A}(x) \vee \bar{\mu}_{A}(y)
\end{aligned}
$$

and $\bar{\mu}_{A}(x a y)=1-\mu_{A}(x a y) \leq 1-\mu_{A}(a)=\bar{\mu}_{A}(a)$. Therefore, $A$ is an intuitionistic fuzzy interior ideal of $S$.

DeFINITION 3.7. Let $A=\left(\mu_{A}, \gamma_{A}\right)$ be an IFS in $S$ and let $\alpha \in[0,1]$. Then the sets

$$
\mu_{A, \alpha}^{\geq}:=\left\{x \in S: \mu_{A}(x) \geq \alpha\right\}, \quad \gamma_{A, \alpha}^{\leq}:=\left\{x \in S: \gamma_{A}(x) \leq \alpha\right\}
$$

are called a $\mu$-level $\alpha$-cut and a $\gamma$-level $\alpha$-cut of $A$, respectively.

THEOREM 3.8. If an IFS $A=\left(\mu_{A}, \gamma_{A}\right)$ in $S$ is an intuitionistic fuzzy interior ideal of $S$, then the $\mu$-level $\alpha$-cut $\mu_{A, \alpha}^{\geq}$and $\gamma$-level $\alpha$-cut $\gamma_{A, \alpha}^{\leq}$of $A$ are interior ideals of $S$ for every $\alpha \in \operatorname{Im}\left(\mu_{A}\right) \cap \operatorname{Im}\left(\gamma_{A}\right) \subseteq[0,1]$.

Proof. Let $\alpha \in \operatorname{Im}\left(\mu_{A}\right) \cap \operatorname{Im}\left(\gamma_{A}\right) \subseteq[0,1]$ and let $x, y \in \mu_{A, \alpha}^{2}$. Then $\mu_{A}(x) \geq \alpha$ and $\mu_{A}(y) \geq \alpha$. It follows from (IF1) that

$$
\mu_{A}(x y) \geq \mu_{A}(x) \wedge \mu_{A}(y) \geq \alpha \quad \text { so that } x y \in \mu_{A, \alpha}^{\geq}
$$

If $x, y \in \gamma_{A, \alpha}^{\leq}$, then $\gamma_{A}(x) \leq \alpha$ and $\gamma_{A}(y) \leq \alpha$, and so

$$
\gamma_{A}(x y) \leq \gamma_{A}(x) \vee \gamma_{A}(y) \leq \alpha, \quad \text { that is, } x y \in \gamma_{A, \alpha}^{\leq} .
$$

Hence $\mu_{A, \alpha}^{2}$ and $\gamma_{A, \alpha}^{\leq}$are subsemigroups of $S$. Now let $x, y \in S$ and $a \in \mu_{A, \alpha}^{2}$. Then $\mu_{A}(x a y) \geq \mu_{A}(a) \geq \alpha$ and so $x a y \in \mu_{A, \alpha}^{\geq}$. If $a \in \gamma_{A, \alpha}^{\leq}$, then $\gamma_{A}(x a y) \leq \gamma_{A}(a) \leq \alpha$ and thus $x a y \in \gamma_{A, \alpha}^{\leq}$. Therefore $\mu_{A, \alpha}^{\geq}$and $\gamma_{A, \alpha}^{\leq}$are interior ideals of $S$.

THEOREM 3.9. Let $A=\left(\mu_{A}, \gamma_{A}\right)$ be an IFS in $S$ such that the nonempty sets $\mu_{A, \alpha}^{2}$ and $\gamma_{A, \alpha}^{\leq}$are interior ideals of $S$ for all $\alpha \in[0,1]$. Then $A=\left(\mu_{A}, \gamma_{A}\right)$ is an intuitionistic fuzzy interior ideal of $S$.

Proof. Let $\alpha \in[0,1]$ and suppose that $\mu_{A, \alpha}^{\geq}(\neq \varnothing)$ and $\gamma_{A, \alpha}^{\leq}(\neq \varnothing)$ are interior ideals of $S$. We must show that $A=\left(\mu_{A}, \gamma_{A}\right)$ satisfies conditions (IF1)-(IF4). If condition (IF1) is false, then there exist $x_{0}, y_{0} \in S$ such that $\mu_{A}\left(x_{0} y_{0}\right)<\mu_{A}\left(x_{0}\right) \wedge \mu_{A}\left(y_{0}\right)$. Taking

$$
\alpha_{0}:=\frac{1}{2}\left(\mu_{A}\left(x_{0} y_{0}\right)+\mu_{A}\left(x_{0}\right) \wedge \mu_{A}\left(y_{0}\right)\right)
$$

we have $\mu_{A}\left(x_{0} y_{0}\right)<\alpha_{0}<\mu_{A}\left(x_{0}\right) \wedge \mu_{A}\left(y_{0}\right)$. It follows that $x_{0}, y_{0} \in \mu_{A, \alpha_{0}}^{\geq}$and $x_{0} y_{0} \notin$ $\mu_{A, \alpha_{0}}^{\geq}$, which is a contradiction. Hence condition (IF1) is true. The proof of other conditions are similar to the case (IF1), we omit the proof. 
THEOREM 3.10. Let $M$ be an interior ideal of $S$ and let $A=\left(\mu_{A}, \gamma_{A}\right)$ be an IFS in $S$ defined by

$$
\mu_{A}(x):=\left\{\begin{array}{ll}
\alpha_{0} & \text { if } x \in M, \\
\alpha_{1} & \text { otherwise }
\end{array} \quad \gamma_{A}(x):= \begin{cases}\beta_{0} & \text { if } x \in M, \\
\beta_{1} & \text { otherwise },\end{cases}\right.
$$

for all $x \in S$ and $\alpha_{i}, \beta_{i} \in[0,1]$ such that $\alpha_{0}>\alpha_{1}, \beta_{0}<\beta_{1}$, and $\alpha_{i}+\beta_{i} \leq 1$ for $i=0,1$. Then $A=\left(\mu_{A}, \gamma_{A}\right)$ is an intuitionistic fuzzy interior ideal of $S$ and $\mu_{A, \alpha_{0}}^{\geq}=M=\gamma_{A, \beta_{0}}^{\leq}$.

Proof. Let $x, y \in S$. If anyone of $x$ and $y$ does not belong to $M$, then

$$
\begin{aligned}
& \mu_{A}(x y) \geq \alpha_{1}=\mu_{A}(x) \wedge \mu_{A}(y), \\
& \gamma_{A}(x y) \leq \beta_{1}=\gamma_{A}(x) \vee \gamma_{A}(y) .
\end{aligned}
$$

Other cases are trivial, and we omit the proof. Hence $A=\left(\mu_{A}, \gamma_{A}\right)$ is an intuitionistic fuzzy subsemigroup of $S$. Now let $x, y, a \in S$. If $a \notin M$, then $\mu_{A}(x a y) \geq \alpha_{1}=\mu_{A}(a)$ and $\gamma_{A}(x a y) \leq \beta_{1}=\gamma_{A}(a)$. Assume that $a \in M$. Since $M$ is an interior ideal of $S$, it follows that $x a y \in M$. Hence $\mu_{A}(x a y)=\alpha_{0}=\mu_{A}(a)$ and $\gamma_{A}(x a y)=\beta_{0}=\gamma_{A}(a)$. Therefore $A=\left(\mu_{A}, \gamma_{A}\right)$ is an intuitionistic fuzzy interior ideal of $S$. Obviously $\mu_{A, \alpha_{0}}^{\geq}=$ $M=\gamma_{A, \beta_{0}}^{\leq}$.

COROLLARY 3.11. Let $\chi_{M}$ be the characteristic function of an interior ideal $M$ of $S$. Then the IFS $\tilde{M}=\left(\chi_{M}, \bar{\chi}_{M}\right)$ is an intuitionistic fuzzy interior ideal of $S$.

THEOREM 3.12. If an IFS $A=\left(\mu_{A}, \gamma_{A}\right)$ is an intuitionistic fuzzy interior ideal of $S$, then

$$
\begin{aligned}
& \mu_{A}(x):=\sup \left\{\alpha \in[0,1] \mid x \in \mu_{A, \alpha}^{\geq}\right\}, \\
& \gamma_{A}(x):=\inf \left\{\alpha \in[0,1] \mid x \in \gamma_{A, \alpha}^{\leq}\right\},
\end{aligned}
$$

for all $x \in S$.

Proof. Let $\delta:=\sup \left\{\alpha \in[0,1] \mid x \in \mu_{A, \alpha}^{2}\right\}$ and let $\varepsilon>0$ be given. Then $\delta-\varepsilon<\alpha$ for some $\alpha \in[0,1]$ such that $x \in \mu_{A, \alpha}^{2}$. It follows that $\delta-\varepsilon<\mu_{A}(x)$ so that $\delta \leq \mu_{A}(x)$ since $\varepsilon$ is arbitrary. We now show that $\mu_{A}(x) \leq \delta$. Let $\mu_{A}(x)=\beta$. Then $x \in \mu_{A, \beta}^{\geq}$and so

$$
\beta \in\left\{\alpha \in[0,1] \mid x \in \mu_{A, \alpha}^{2}\right\} .
$$

Hence $\mu_{A}(x)=\beta \leq \sup \left\{\alpha \in[0,1] \mid x \in \mu_{A, \alpha}^{\geq}\right\}=\delta$. Therefore

$$
\mu_{A}(x)=\delta=\sup \left\{\alpha \in[0,1] \mid x \in \mu_{A, \alpha}^{\geq}\right\} .
$$

Now let $\eta=\inf \left\{\alpha \in[0,1] \mid x \in \gamma_{A, \alpha}^{\leq}\right\}$. Then

$$
\inf \left\{\alpha \in[0,1] \mid x \in \gamma_{A, \alpha}^{\leq}\right\}<\eta+\varepsilon \quad \text { for any } \varepsilon<0,
$$

and so $\alpha<\eta+\varepsilon$ for some $\alpha \in[0,1]$ with $x \in \gamma_{A, \alpha}^{\leq}$. Since $\gamma_{A}(x) \leq \alpha$ and $\varepsilon$ is arbitrary, it follows that $\gamma_{A}(x) \leq \eta$. To prove $\gamma_{A}(x) \geq \eta$, let $\gamma_{A}(x)=\zeta$. Then $x \in \gamma_{A, \zeta}^{\leq}$and thus $\zeta \in\left\{\alpha \in[0,1] \mid x \in \gamma_{A, \alpha}^{\leq}\right\}$. Hence

$$
\inf \left\{\alpha \in[0,1] \mid x \in \gamma_{A, \alpha}^{\leq}\right\} \leq \zeta, \quad \text { that is, } \eta \leq \zeta=\gamma_{A}(x) .
$$


Consequently,

$$
\gamma_{A}(x)=\eta=\inf \left\{\alpha \in[0,1] \mid x \in \gamma_{A, \alpha}^{\leq}\right\} .
$$

This completes the proof.

THEOREM 3.13. Let $\left\{C_{\alpha} \mid \alpha \in \Lambda\right\}$ be a collection of interior ideals of $S$ such that

(i) $S=\cup_{\alpha \in \Lambda} C_{\alpha}$,

(ii) $\beta>\alpha$ if and only if $C_{\beta} \subset C_{\alpha}$ for all $\beta, \alpha \in \Lambda$.

Then an IFS $A=\left(\mu_{A}, \gamma_{A}\right)$ in $S$ defined by

$$
\begin{aligned}
& \mu_{A}(x):=\sup \left\{\alpha \in \Lambda \mid x \in C_{\alpha}\right\}, \\
& \gamma_{A}(x):=\inf \left\{\alpha \in \Lambda \mid x \in C_{\alpha}\right\},
\end{aligned}
$$

for all $x \in S$, is an intuitionistic fuzzy interior ideal of $S$.

Proof. Following Theorem 3.9, it is sufficient to show that the nonempty level sets $\mu_{A, \alpha}^{\geq}$and $\gamma_{A, \alpha}^{\leq}$are interior ideals of $S$ for every $\alpha \in[0,1]$. In order to prove that $\mu_{A, \alpha}^{\geq}(\neq \varnothing)$ is an interior ideal, we have the following two cases:

(i) $\alpha=\sup \{\delta \in \Lambda \mid \delta<\alpha\}$ and

(ii) $\alpha \neq \sup \{\delta \in \Lambda \mid \delta<\alpha\}$.

Case (i) implies that

$$
x \in \mu_{A, \alpha}^{\geq} \Longleftrightarrow x \in C_{\delta} \quad \forall \delta<\alpha \Longleftrightarrow x \in \cap_{\delta<\alpha} C_{\delta},
$$

so that $\mu_{A, \alpha}^{\geq}=\cap_{\delta<\alpha} C_{\delta}$, which is an interior ideal of $S$. For the case (ii), we claim that $\mu_{A, \alpha}^{\geq}=\cup_{\delta \geq \alpha} C_{\delta}$. If $x \in \cup_{\delta \geq \alpha} C_{\delta}$, then $x \in C_{\delta}$ for some $\delta \geq \alpha$. It follows that $\mu_{A}(x) \geq \delta \geq \alpha$, so that $x \in \mu_{A, \alpha}^{\geq}$. This proves that $\cup_{\delta \geq \alpha} C_{\delta} \subseteq \mu_{A, \alpha}^{\geq}$. Now assume that $x \notin \cup_{\delta \geq \alpha} C_{\delta}$. Then $x \notin C_{\delta}$ for all $\delta \geq \alpha$. Since $\alpha \neq \sup \{\delta \in \Lambda \mid \delta<\alpha\}$, there exists $\varepsilon>0$ such that $(\alpha-\varepsilon, \alpha) \cap \Lambda=\varnothing$. Hence $x \notin C_{\delta}$ for all $\delta>\alpha-\varepsilon$, which means that if $x \in C_{\delta}$ then $\delta \leq \alpha-\varepsilon$. Thus $\mu_{A}(x) \leq \alpha-\varepsilon<\alpha$, and so $x \notin \mu_{A, \alpha}^{\geq}$. Therefore $\mu_{A, \alpha}^{\geq} \subseteq \cup_{\delta \geq \alpha} C_{\delta}$, and thus $\mu_{A, \alpha}^{\geq}=\cup_{\delta \geq \alpha} C_{\delta}$ which is an interior ideal of $S$. Next we prove that $\gamma_{A, \alpha}^{\leq}(\neq \varnothing)$ is an interior ideal of $S$ for all $\alpha \in[0,1]$. We consider the following two cases:

(iii) $\beta=\inf \{\delta \in \Lambda \mid \beta<\delta\}$ and

(iv) $\beta \neq \inf \{\delta \in \Lambda \mid \beta<\delta\}$.

For the case (iii) we have

$$
x \in \gamma_{A, \beta}^{\leq} \Longleftrightarrow x \in C_{\delta} \quad \forall \beta<\delta \Longleftrightarrow x \in \cap_{\beta<\delta} C_{\delta},
$$

and hence $\gamma_{A, \beta}^{\leq}=\cap_{\beta<\delta} C_{\delta}$ which is an interior ideal of $S$. For the case (iv), there exists $\varepsilon>0$ such that $(\beta, \beta+\varepsilon) \cap \Lambda=\varnothing$. We show that $\gamma_{A, \beta}^{\leq}=\cup_{\beta \geq \delta} C_{\delta}$. If $x \in \cup_{\beta \geq \delta} C_{\delta}$, then $x \in C_{\delta}$ for some $\beta \geq \delta$. It follows that $\gamma_{A}(x) \leq \delta \leq \beta$ so that $x \in \gamma_{A, \beta}^{\leq}$. Hence $\cup_{\beta \geq \delta} C_{\delta} \subseteq$ $\gamma_{A, \beta}^{\leq}$. Conversely, if $x \notin \cup_{\beta \geq \delta} C_{\delta}$ then $x \notin C_{\delta}$ for all $\delta \leq \beta$, which implies that $x \notin C_{\delta}$ for all $\delta<\beta+\varepsilon$, that is, if $x \in C_{\delta}$ then $\delta \geq \beta+\varepsilon$. Thus $\gamma_{A}(x) \geq \beta+\varepsilon>\beta$, that is, $x \notin \gamma_{A, \beta}^{\leq}$. Therefore $\gamma_{A, \beta}^{\leq} \subseteq \cup_{\beta \geq \delta} C_{\delta}$ and consequently $\gamma_{A, \beta}^{\leq}=\cup_{\beta \geq \delta} C_{\delta}$ which is an interior ideal of $S$. This completes the proof.

THEOREM 3.14. An IFS $A=\left(\mu_{A}, \gamma_{A}\right)$ is an intuitionistic fuzzy interior ideal of $S$ if and only if the fuzzy sets $\mu_{A}$ and $\bar{\gamma}_{A}$ are fuzzy interior ideals of $S$. 
Proof. Let $A=\left(\mu_{A}, \gamma_{A}\right)$ be an intuitionistic fuzzy interior ideal of $S$. Then clearly $\mu_{A}$ is a fuzzy interior ideal of $S$. Let $x, a, y \in S$. Then

$$
\begin{aligned}
\bar{\gamma}_{A}(x y) & =1-\gamma_{A}(x y) \geq 1-\gamma_{A}(x) \vee \gamma_{A}(y) \\
& =\left(1-\gamma_{A}(x)\right) \wedge\left(1-\gamma_{A}(y)\right)=\bar{\gamma}_{A}(x) \wedge \bar{\gamma}_{A}(y), \\
\bar{\gamma}_{A}(x a y) & =1-\gamma_{A}(x a y) \geq 1-\gamma_{A}(a)=\bar{\gamma}_{A}(a) .
\end{aligned}
$$

Hence $\bar{\gamma}_{A}$ is a fuzzy interior ideal of $S$.

Conversely, suppose that $\mu_{A}$ and $\bar{\gamma}_{A}$ are fuzzy interior ideals of $S$. Let $a, x, y \in S$. Then

$$
\begin{aligned}
1-\gamma_{A}(x y) & =\bar{\gamma}_{A}(x y) \geq \bar{\gamma}_{A}(x) \wedge \bar{\gamma}_{A}(y) \\
& =\left(1-\gamma_{A}(x)\right) \wedge\left(1-\gamma_{A}(y)\right) \\
& =1-\gamma_{A}(x) \vee \gamma_{A}(y), \\
1-\gamma_{A}(x a y) & =\bar{\gamma}_{A}(x a y) \geq \bar{\gamma}_{A}(a)=1-\gamma_{A}(a),
\end{aligned}
$$

which imply that $\gamma_{A}(x y) \leq \gamma_{A}(x) \vee \gamma_{A}(y)$ and $\gamma_{A}(x a y) \leq \gamma_{A}(a)$. This completes the proof.

COROLLARY 3.15. An IFS $A=\left(\mu_{A}, \gamma_{A}\right)$ is an intuitionistic fuzzy interior ideal of $S$ if and only if $\square A=\left(\mu_{A}, \bar{\mu}_{A}\right)$ and $\diamond A=\left(\bar{\gamma}_{A}, \gamma_{A}\right)$ are intuitionistic fuzzy interior ideals of $S$.

Proof. The proof is straightforward by Theorem 3.14.

Let $f$ be a map from a set $X$ to a set $Y$. If $A=\left(\mu_{A}, \gamma_{A}\right)$ and $B=\left(\mu_{B}, \gamma_{B}\right)$ are IFSs in $X$ and $Y$, respectively, then the preimage of $B$ under $f$, denoted by $f^{-1}(B)$, is an IFS in $X$ defined by

$$
f^{-1}(B)=\left(f^{-1}\left(\mu_{B}\right), f^{-1}\left(\gamma_{B}\right)\right), \text { where } f^{-1}\left(\mu_{B}\right)=\mu_{B}(f) .
$$

THEOREM 3.16. Let $f: S \rightarrow T$ be a homomorphism of semigroups. If $B=\left(\mu_{B}, \gamma_{B}\right)$ is an intuitionistic fuzzy interior ideal of $T$, then the preimage $f^{-1}(B)=\left(f^{-1}\left(\mu_{B}\right), f^{-1}\left(\gamma_{B}\right)\right)$ of $B$ under $f$ is an intuitionistic fuzzy interior ideal of $S$.

Proof. Assume that $B=\left(\mu_{B}, \gamma_{B}\right)$ is an intuitionistic fuzzy interior ideal of $T$ and let $x, y \in S$. Then

$$
\begin{aligned}
f^{-1}\left(\mu_{B}\right)(x y) & =\mu_{B}(f(x y)) \\
& =\mu_{B}(f(x) f(y)) \\
& \geq \mu_{B}(f(x)) \wedge \mu_{B}(f(y)) \\
& =f^{-1}\left(\mu_{B}(x)\right) \wedge f^{-1}\left(\mu_{B}(y)\right), \\
f^{-1}\left(\gamma_{B}\right)(x y) & =\gamma_{B}(f(x y)) \\
& =\gamma_{B}(f(x) f(y)) \\
& \leq \gamma_{B}(f(x)) \vee \gamma_{B}(f(y)) \\
& =f^{-1}\left(\gamma_{B}(x)\right) \vee f^{-1}\left(\gamma_{B}(y)\right) .
\end{aligned}
$$


Hence $f^{-1}(B)=\left(f^{-1}\left(\mu_{B}\right), f^{-1}\left(\gamma_{B}\right)\right)$ is an intuitionistic fuzzy subsemigroup of $S$. For any $a, x, y \in S$, we have

$$
\begin{aligned}
f^{-1}\left(\mu_{B}\right)(x a y) & =\mu_{B}(f(x a y)) \\
& =\mu_{B}(f(x) f(a) f(y)) \\
& \geq \mu_{B}(f(a)) \\
& =f^{-1}\left(\mu_{B}(a)\right), \\
f^{-1}\left(\gamma_{B}\right)(x a y) & =\gamma_{B}(f(x a y)) \\
& =\gamma_{B}(f(x) f(a) f(y)) \\
& \leq \gamma_{B}(f(a)) \\
& =f^{-1}\left(\gamma_{B}(a)\right) .
\end{aligned}
$$

Therefore $f^{-1}(B)=\left(f^{-1}\left(\mu_{B}\right), f^{-1}\left(\gamma_{B}\right)\right)$ is an intuitionistic fuzzy interior ideal of $S$.

ACKNOWLEDGEMENT. The authors are deeply grateful to the referees for the valuable suggestions.

\section{REFERENCES}

[1] K. T. Atanassov, Intuitionistic fuzzy sets, Fuzzy Sets and Systems 20 (1986), no. 1, 87-96. MR 87f:03151. Zbl 631.03040.

[2] _ New operations defined over the intuitionistic fuzzy sets, Fuzzy Sets and Systems 61 (1994), no. 2, 137-142. CMP 1262 464. Zbl 824.04004.

[3] S. M. Hong, Y. B. Jun, and J. Meng, Fuzzy interior ideals in semigroups, Indian J. Pure Appl. Math. 26 (1995), no. 9, 859-863. CMP 1347 533. Zbl 834.20074.

[4] J. M. Howie, An Introduction to Semigroup Theory, Academic Press, London, 1976. MR 57\#6235. Zbl 355.20056.

KyUng Ho Kim: Department of Mathematics, ChunguU National University, ChungJu 380-702, KOREA

E-mail address: ghkim@gukwon. chungju.ac. kr

Young BAe Jun: Department of MATHematics Education, GyeONGSANG NATional UniVERSITY, CHINJU 660-701, KOREA

E-mail address: ybjun@nongae.gsnu.ac.kr 


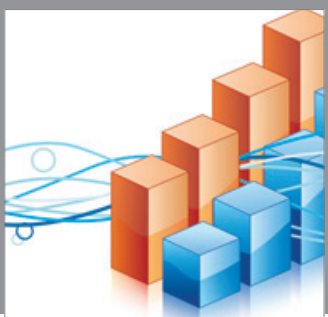

Advances in

Operations Research

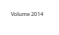

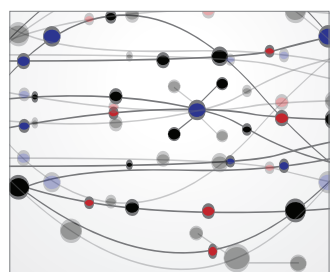

\section{The Scientific} World Journal
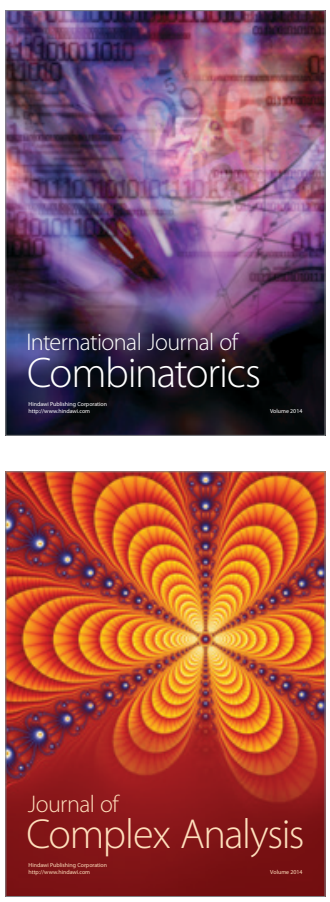

International Journal of

Mathematics and

Mathematical

Sciences
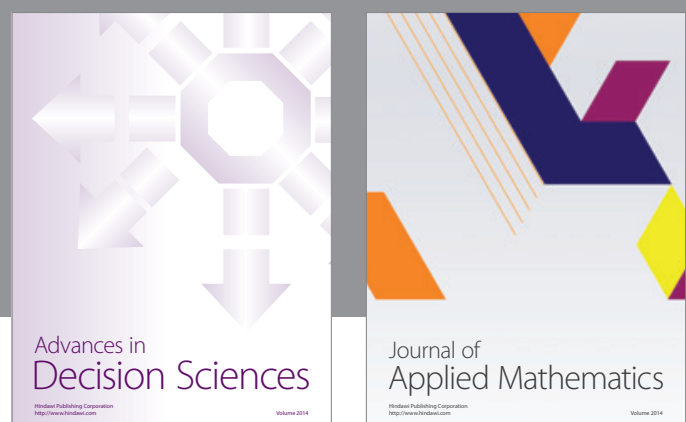

Journal of

Applied Mathematics
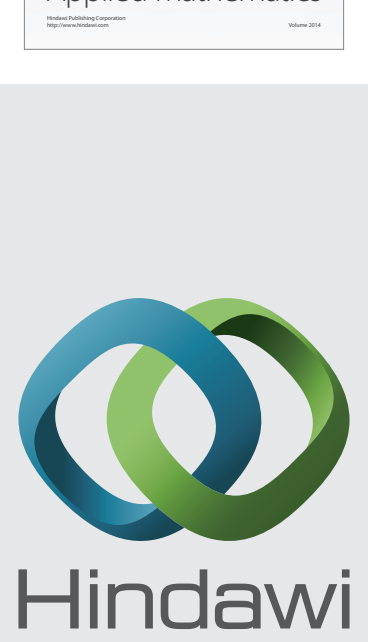

Submit your manuscripts at http://www.hindawi.com
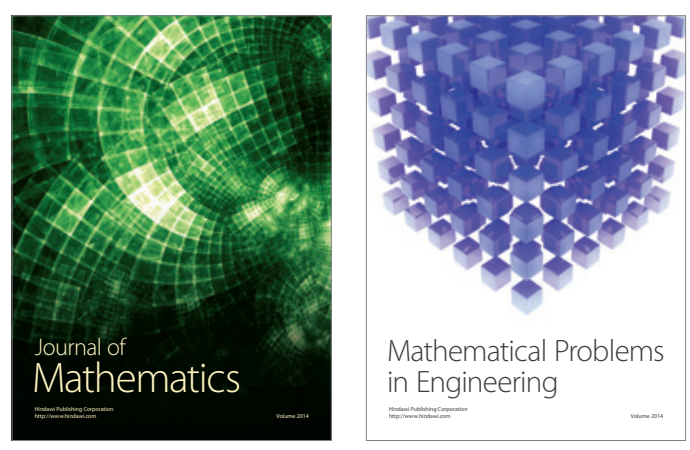

Mathematical Problems in Engineering
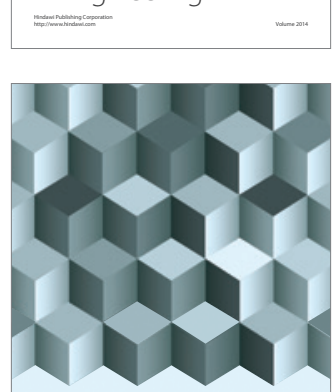

Journal of

Function Spaces
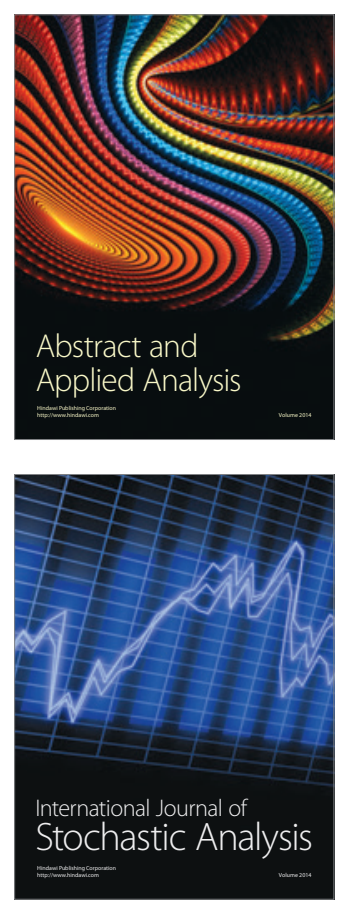

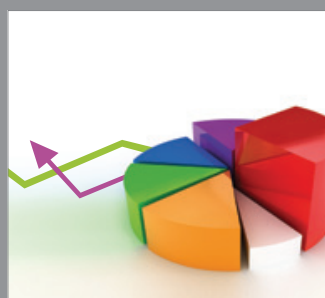

ournal of

Probability and Statistics

Promensencen
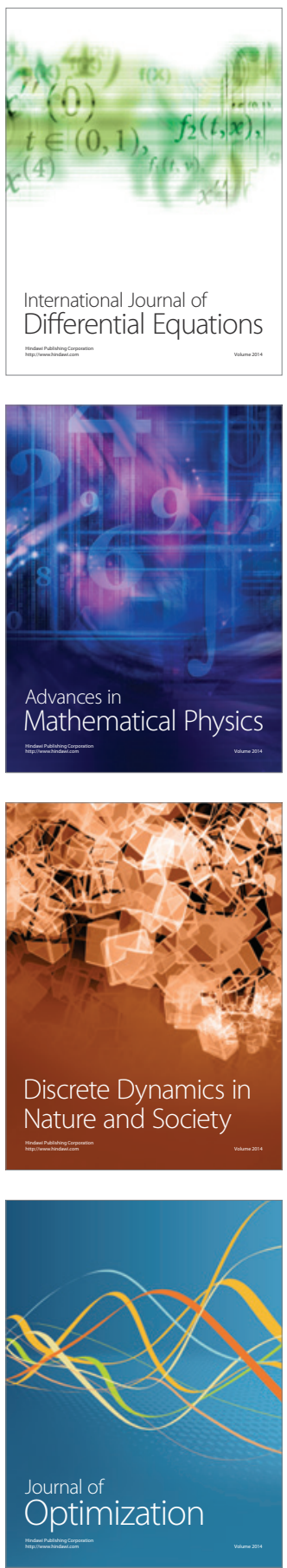